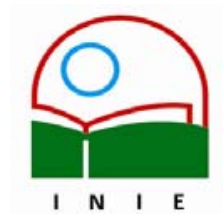

Actualidades Investigativas en Educación

Revista Electrónica publicada por el

Instituto de Investigación en Educación

Universidad de Costa Rica

ISSN 1409-4703

http://revista.inie.ucr.ac.cr

COSTA RICA

\title{
LA POLÍTICA EXTERIOR NORTEAMERICANA DESDE LA ÓPTICA EDUCATIVA DEL VECINO DEL SUR
}

FROM A NEIGHBOR TO THE SOUTH: MEXICAN EDUCATORS AND THEIR TEACHING OF CURRENT UNITED STATES POLICIES

Volumen 7, Número 1

Enero-Abril 2007

pp. 1-22

Este número se publicó el 30 de abril 2007

Timothy G. Cashman

Rene A. Rubio

La revista está indexada en los directorios:

LATINDEX, REDALYC, IRESIE, CLASE, DIALNET, DOAJ, E-REVIST@S,

La revista está incluida en los sitios:

REDIE, RINACE, OEI, MAESTROTECA, HUASCARAN

Los contenidos de este artículo están baj o una licencia Creative Commons 


\title{
LA POLÍTICA EXTERIOR NORTEAMERICANA DESDE LA ÓPTICA EDUCATIVA DEL VECINO DEL SUR \\ FROM A NEIGHBOR TO THE SOUTH: MEXICAN EDUCATORS AND THEIR TEACHING OF CURRENT UNITED STATES POLICIES
}

\section{Timothy G. Cashman Rene A. Rubio ${ }^{2}$}

\begin{abstract}
Resumen: En este estudio los investigadores analizan las percepciones de profesoras, profesores, personal administrativo y estudiantes en una ciudad norteña de México, en relación con la política exterior de los Estados Unidos de Norteamérica. Esta investigación tiene lugar en un momento en que las relaciones entre ambos países son tensas a raíz de la autorización de la construcción del muro fronterizo entre las dos naciones. El objetivo del estudio es generar una mayor comprensión acerca de las recientes políticas estadounidenses, y su impacto en el extranjero. Además, compara y contrasta la perspectiva de profesores y estudiantes mexicanos sobre los mensajes trasmitidos por los medios de comunicación norteamericanos. Finalmente, se presenta una serie de recomendaciones en las que se enfatiza en la construcción de puentes de entendimiento en un tiempo en el que al parecer los muros van ganando la batalla.
\end{abstract}

Palabras clave: POLÍTICA EXTERIOR ESTADOUNIDENSE, RELACIÓN ENTRE MÉXICO Y LOS ESTADOS UNIDOS, CIENCIAS SOCIALES.

\begin{abstract}
Researchers analyze the perceptions and pedagogies of educators and students in northern Mexico with regard to United States foreign policies. The key objective of the research is to provide additional insight to the impact abroad of recent actions taken by the United States government. Furthermore, this study compares and contrasts the viewpoints of Mexican educators and students with coverage provided by the mainstream United States media and United States educational systems. Educators and future teachers in both the United States and Mexico benefit from broader understandings of educators' roles and students' perceptions in a bi-national community.
\end{abstract}

Keywords: US FOREIGN POLICY, MEXICO-USA RELATIONSHIP, SOCIAL SCIENCES.

\begin{abstract}
${ }^{1}$ El grado de Doctor fue obtenido en Washington State University. Sus estudios de Licenciatura y Maestría, ambos dentro del ámbito educativo los obtuvo en la University of Northern lowa y University of New México respectivamente. Actualmente trabaja en el Colegio de Educación de la Universidad de Texas en el Paso.

${ }^{2}$ Candidato a Doctor en la Universidad de Texas en el Paso. Sus estudios de Licenciatura y Maestría fueron obtenidos en al Universidad de las Américas, Puebla, México. Actualmente trabaja como Assistant Intstructor en el Colegio de Educación en la Universidad de Texas en el Paso.
\end{abstract}

Correo electrónico: rarubio2@utep.edu

Artículo recibido: 28 de octubre, 2006

Aprobado: 25 de abril, 2007 


\section{Introducción}

Sui generis es el concepto que cualitativamente mejor describe el tiempo y el espacio a los que hace referencia la presente investigación. Esta tiene lugar en un tiempo en que la inminente construcción de un frío muro evidencia la calidez entre "vecinos distantes". Este hecho histórico hace patente, más que nunca, que los 3147 kilómetros de frontera compartida siguen siendo la puerta amplia para la inmigración ilegal, la cara más conocida de México en Norteamérica y la consecuente discriminación como práctica recurrente de los Estados Unidos en contra de la población mexicana.

El presente estudio tuvo lugar en la ciudad de Chihuahua, México. Cabe mencionar que esta localidad es asimismo capital del Estado mexicano de Chihuahua, cuya extensión de 250,000 km. cuadrados lo define como el Estado más grande de México. Esta comunidad chihuahuense se constituyó en contexto de estudio, por considerarse que los 350 Km. que la separan del Paso, Texas, USA. La hacen ser un escenario de investigación propicio por su relativo aislamiento tanto de los medios de comunicación como del sistema educativo estadounidenses. Su población está constituida por 700,000 habitantes según información del Instituto Nacional de Estadística Geografía e Informática (2000).

Los investigadores al frente de este estudio están afiliados a una universidad ubicada en el suroeste de los Estados Unidos. Esta institución cuenta con una población cercana a los 20,000 estudiantes, de los cuales el $80 \%$ es mexicano o de origen méxico-americano. Esta estadística correspondiente a los estudiantes mexicanos dentro de la universidad refleja también las características demográficas de la comunidad en que esta universidad se localiza en el lado estadounidense de la línea fronteriza. Los investigadores viven en una zona metropolitana constituida por aproximadamente 800,000 habitantes en el lado estadounidense. Hacia el sur compartiendo la mancha urbana, se encuentra una ciudad de 1.8 millones de personas, separada físicamente de la primera por cinco puentes internacionales.

Tal y como se explica a continuación, esta investigación consistió en la detección perceptual y actitudinal tanto de profesoras, profesores como de estudiantes mexicanos de ciencias sociales en relación con la política exterior de los Estados Unidos y su impacto en la comunidad global. Esta investigación tiene lugar en un momento en que las relaciones entre ambos países son tensas a raíz de la autorización de la construcción del muro fronterizo entre las dos naciones. Para tales fines, tanto personal directivo como docente de los cursos de ciencias sociales fueron entrevistados en dos escuelas públicas de la ciudad de Chihuahua. En ambas instituciones, estudiantes, personal administrativo, docente y de 
apoyo evidenciaron en todo momento un profundo sentimiento de pertenencia y orgullo por sus instituciones.

\section{Objetivo}

Los tiempos de guerra y conflicto constituyen elementos propicios en que las perspectivas de otros, no directamente involucrados en la violencia se presentan como fundamentos imperativos para una comprensión holística de los fenómenos sociales. Regularmente aquellos no directamente involucrados de forma directa en la guerra y en el conflicto ofrecen una perspectiva fresca y rica. Por el simple hecho, de ser una nación en guerra, los Estados Unidos deben promocionar un mayor y mejor conocimiento del tópico, a partir de "la plenitud de narraciones al alcance" (Bender, 2002, p.19). Como investigadores percibimos una necesidad manifiesta por compartir las perspectivas de educadores y estudiantes de un país vecino, que por razones históricas cuenta con una dinámica de interconexión económica y cultural con los Estados Unidos como ningún otro país en el mundo.

El objetivo del estudio es generar una mayor comprensión acerca de las recientes políticas estadounidenses, y su impacto en el extranjero. La perspectiva de México es particularmente importante, ya que es un país que a través de su gente se presenta como extremadamente independiente, pero a la vez económica y culturalmente interconectado con los Estados Unidos de Norteamérica.

Veintisiete participantes de ambos sexos, tanto docentes como administrativos, pertenecientes a dos instituciones fueron entrevistados para lograr los objetivos de este estudio. Su participación fue solicitada con el objetivo -entre otros- de lograr una mejor comprensión de las diferencias discursivas en las ciencias sociales, por ser esta disciplina en la cual se promueve el análisis de aspectos internacionales, entre escuelas chihuahuenses y escuelas en los Estados Unidos. Las secciones diferenciadas de participantes incluyeron a profesores de últimos grados de primaria y secundaria.

Este estudio provee un mayor cúmulo de información acerca del impacto de la política exterior de los Estados Unidos desde la óptica de comunidades educativas mexicanas, políticas que incluyen los más recientes acontecimientos. Por esta razón, (5) administrativos, (22) profesores fueron entrevistados: catrorce mujeres y ocho varones, para ampliar así el alcance de los resultados. El personal administrativo incluyó a tres hombres y dos mujeres. Ellos fueron quienes seleccionaron a los profesores entrevistados en ambas instituciones. Además, cinco grupos de estudiantes fueron formalmente observados. Este trabajo compara 
y contrasta las perspectivas de los educadores mexicanos con aquellas opiniones vertidas a la audiencia norteamericana por parte de los medios de comunicación estadounidenses. Las siguientes preguntas orientaron esta investigación cualitativa:

- ¿Considera importante la guerra promovida por los Estados Unidos en Irak dentro de sus discusiones en clase?

- ¿Existe facilidad y apertura al dialogo existe en su clase?

- ¿Cuál es la diferencia ideológica más recurrente entre México y los Estados Unidos que surge a raíz de sus discusiones acerca de la política exterior estadounidense?

- ¿Por qué otros, y en particular la sociedad norteamericana, deberían estar informados acerca de las opiniones derivadas de las clases de ciencias sociales impartidas en aulas chihuahuenses?

\section{Panorama de la educación en México}

A pesar del largo historial prehispánico y colonial mexicano, el primer esfuerzo de consolidación de la educación en torno a una visión nacional fue efectuado por el Presidente Benito Juárez. Durante su mandato, Juárez promovió el establecimiento de las escuelas públicas para los niveles de primaria, secundaria y profesional, hecho que tuvo lugar diez años después de la guerra con los Estados Unidos y la consecuente firma del tratado de Guadalupe Hidalgo en 1848. El plan curricular de 1848 incluía eminentemente estudios de moralidad, democracia, y temáticas acerca del incipiente derecho mexicano (Rippberger \& Staudt, 1993). Este plan estaba fuertemente permeado por un énfasis en la integración de valores sociales enfocados a la creación de la constante prioridad de asegurar la identidad nacional (Perez, 2006).

Los tradicionales cambios revolucionarios mexicanos hicieron que la Constitución de 1917 marcara el inicio de la educación moderna en este país. La educación garantizada por el Estado estuvo sustentada en los principios sociopolíticos de libertad, obligatoriedad y universalidad (Pescador, 1985). La misión fundamental de la escuela estaba enfocada hacia la promoción de la unidad nacional. Por ello las escuelas privadas y religiosas debieron operar bajo la supervisión del Estado. En 1921 aparece en escena la Secretaría de Educación Pública, cuyo cometido contemplaba la supervisión de las escuelas de todo tipo, incluyendo por supuesto aquellas de financiamiento privado y religioso.

Durante el lapso comprendido en los años 1960 y 1970, la Secretaría de Educación Pública (SEP) definió un esquema curricular tendiente a la creación de un rol activo por parte 
del estudiante en su propio proceso de aprendizaje. La pedagogía predominante en este esfuerzo promovía una mayor creatividad, fundamento científico y pensamiento crítico dentro del proceso educativo. Cabe hacer mención que el gobierno ha continuado la promoción de la modernización de la educación que incluye tecnología educativa aplicable en el aula. Sin embargo algunos de los objetivos de largo alcance permanecen aún como "objetivos idealistas" por carecer de adecuados esquemas de financiamiento (Rippberger \& Staudt, 2003).

En nuestros días la SEP, como instancia federal, es la figura de mayor presencia y autoridad en el ámbito educativo mexicano. Dentro de las expectativas de este sistema están el logro y la congruencia de contenidos educativos aplicables en los ámbitos rurales y urbanos, así como servir de enlace con las secretarías federales de agricultura, salud y otras de asistencia social. Sin embargo, el fundamento básico de la SEP está orientado hacia la promoción permanente del sentimiento de identidad nacional y patriotismo (Erickson, 2003). La educación básica mexicana pretende que los estudiantes cuenten con los conocimientos fundamentales y las habilidades intelectuales para su eficaz inserción en la sociedad (Sistema Educativo de los Estados Unidos Mexicanos, 2004). Como una consecuencia lógica de lo anterior, uno de los objetivos principales de las escuelas públicas consiste en la promoción de un sentimiento de conciencia social a través de las ciencias sociales.

Los resultados de la investigación indican que los conceptos de solidaridad y equidad están íntimamente conectados dentro de la educación mexicana (Levinson, 1998). Los uniformes escolares han sido establecidos en este mismo sentido con el objetivo de eliminar posibles causas de discriminación entre los estudiantes. Con base en información vertida por autoridades educativas, se puede afirmar que la disminución de diferencias en el vestir de los estudiantes sirve para minimizar la percepción de diferencias económicas y sociales en la población estudiantil. Toda la planta educativa hace un marcado énfasis en la necesidad que deben tener los estudiantes para trabajar en la consecución del fin común. En México, el proceso de construcción de nación y el proceso de construcción de la identidad nacional permanecen como un sólido imperativo, especialmente por estar México en la constante sombra de su poderoso vecino del norte (Levinson, 1998).

Tanto dentro del discurso oficial como dentro de la cultura estudiantil es perceptible un sentimiento de solidaridad colectiva. En este sentido Levinson (1998) identificó la ausencia de polarización de la escuela secundaria mexicana, y define que la estructura educativa y sus acciones específicas promueven de alguna forma el desarrollo de compasión y solidaridad social. 
El análisis del autor previamente citado sugiere que la instrucción fundamentada en tópicos relativos a la solidaridad y equidad por parte del docente, aún en grupos heterogéneos tiene mucho que ver con la ausencia de divisionismo social. Hampton, Ligouri, and Rippberger (2003) han descrito sus propias experiencias multiculturales en investigaciones previas efectuadas en escuelas pertenecientes a una populosa ciudad fronteriza del Estado de Chihuahua. Dichos autores analizaron el impacto de las relaciones de poder y globalización en la educación, así como la estructura social de comunidades laborales de esta ciudad fronteriza. De particular importancia resulta mencionar que dentro de sus conclusiones está el hecho de que la educación y el aprendizaje tienen lugar en esta zona bajo una dolorosa escasez de material de todo tipo (Hampton, Liguori, Rippberger, 2003).

Históricamente, las relaciones de género implícitas en la estructura social han afectado las oportunidades laborales de la mujer, muchas de las cuales han optado por la enseñanza como una opción de desarrollo profesional (Jelin, 1990; Howell, 1997). Ahora, en el norte de México, tal y como sucede en otras partes del país, la recia figura de la mujer norteña juega un rol vital en la función educativa de la escuela secundaria. La participación de la mujer en esta investigación fue determinante, ya que en todo momento evidenciaron un marcado interés en la educación, en los fines propios de esta investigación, así como en la propuesta para la construcción de mecanismos permanentes de colaboración con instituciones de los Estados Unidos.

\section{Análisis crítico del sistema educativo mexicano}

En este apartado se pretende la descripción general acerca de la operación pedagógica, didáctica y administrativa prevaleciente en la educación secundaria mexicana, con el objeto de proporcionar el marco operativo de este sistema de educación en el cual se ubican las escuelas motivo de estudio. Entre las mayores preocupaciones del sistema educativo mexicano se halla el desigual otorgamiento de recursos y la distribución del ingreso, especialmente entre las comunidades indígenas. Al respecto uno de los más respectados académicos mexicanos del siglo Veinte, Jaime Torres Bodet, destaca que "el progreso como nación no debe ser el progreso de una minoría privilegiada, sino de todos los ciudadanos, yo repito, todos los ciudadanos, sin la discriminación que resulta incompatible con el libre, justo e informado ejercicio democrático" (1961, p. 2).

Los procesos oficiales de educación en México se encuentran en un periodo de transición. Las reformas efectuadas en la materia en 1993 estuvieron focalizadas en la 
educación secundaria. En este sentido, el Articulo 30 de la Constitución obligó en su momento al aparato gubernamental a generar estrategias para incrementar el número de escuelas del nivel secundaria en todo el país. Con esta obligación se pretendió dar cumplimiento a lo establecido en el Programa para la Modernización Educativa (1989-1994), en el sentido de asegurar la permanencia educativa de los estudiantes que al terminar la educación primaria tienen la obligación y el derecho de continuar su educación en la secundaria. Por esta causa, en la actualidad grupos de educadores visitan regularmente las escuelas primarias con el objetivo de promocionar la permanencia educativa y la importancia de continuar los estudios en la educación secundaria y más allá (Andrade de Herrara, 1996).

Otro de los componentes importantes de estas reformas educativas fue la descentralización educativa, es decir, la transición del ámbito federal al estatal, en cuanto a la responsabilidad de proveer el servicio educativo. Sin embargo, cabe mencionar que la descentralización de la educación es un proceso que aún continúa en México, principalmente en cuestiones relativas al ejercicio presupuestal. En el caso específico de Chihuahua el estado ya planea e implementa sus particulares programas educativos presumiblemente con la intención de contextualizar y resolver las más sentidas necesidades educativas.

A pesar de la relativa libertad en la planeación, aún los secretarios estatales de educación, así como administrativos, profesores y otros colaboradores del sistema tienen que cumplir con requisitos básicos establecidos a nivel federal (Andrade de Herrara, 1996). De esta forma, los actores responsables de la administración de la educación pública se encuentran en el proceso de reajuste dentro y hacia un sistema de mayor autonomía.

Estos cambios estructurales han traído consigo importantes modificaciones en materia de metodología y filosofía de la educación. Existe evidencia de una mayor autonomía en el salón de clase, en donde los profesores deciden y definen temas específicos, promueven el dialogo a través de preguntas abiertas, así como el debate, se facilita asimismo las actividades mediante aprendizaje cooperativo con el propósito de motivar la participación de los alumnos en la construcción de sus propios procesos de aprendizaje.

Algunas escuelas en México están actualmente promocionando una educación crítica que motive el pensamiento reflexivo, y que consecuentemente aliente la consecución de una mayor comprensión de los contenidos instruccionales (Wilcox \& Moreno, 1999). De forma más reciente, sin embargo, los profesores cuentan con una nueva preocupación derivada de la implantación de un nuevo plan curricular nacional y en particular por los propuestos mecanismos de evaluación derivados de este. Cabe hacer mención que estos exámenes nacionales son muy similares a los aplicados en los Estados Unidos. 
La pluriculturalidad es característica fundamental y motivo de orgullo en México, ya que sus "distintas etnias, culturas, comunidades, y grupos comparten derechos y obligaciones comunes en materia económica, política y educativa, a pesar de las -en ocasiones abismales- distinciones en los ámbitos culturales, étnicos y lingüísticos" (Morales \& Caballero, 2002, p. 55). Sin embargo, en muchos sectores de la sociedad mexicana principalmente ubicados en el sur de la república, esta diversidad cultural que lleva implícita la heterogeneidad social mexicana se constituye en uno de los grandes retos por vencer para la consecución de la identidad nacional. Como resultado de la anterior "problemática", algunos de los proyectos nacionales han sido expresamente creados para "eliminar las idiosincrasias culturales de la nación" (Morales \& Caballero, 2002, p. 56). Al respecto, la población indígena sostiene que, más allá de la retórica gubernamental, debería existir un mayor respeto por la diversidad cultural con la finalidad de avanzar en la eliminación de la discriminación y la injusticia social. Así, en lugar de "borrar" del mapa social las diversas idiosincrasias diseminadas en la nación, nuevos educadores con una visión crítica y progresista deberían ser insertados en el sistema educativo. Estos nuevos educadores deberían contar con un nuevo esquema de habilidades didácticas que no se limitaran a la transmisión mecánica de conocimientos y al mantenimiento de la inercia del sistema, ni a legitimar la visión e historia "oficial" mexicanas y que finalmente tampoco reprodujeran practicas desalentadoras de la conciencia social pluriétnica. Existe un reclamo por una nueva concepción de la educación indígena, la cual reconozca "la filosofía universal y los principios científicos, y que al mismo tiempo, se fundamente en las tradiciones históricas $y$ conocimiento cultural propio a través del uso de la lengua materna" (Morales \& Caballero, 2002, p. 56).

Otro de los puntos medulares consiste en la gran necesidad por alcanzar un mayor entendimiento sobre el rol de la mujer mexicana en la educación (Howell, 1997; Jelin, 1990). Con base en un estudio realizado por Howell (1997) relativo a las profesoras Oaxaqueñas, menciona que las mujeres dedicadas a la enseñanza decidieron por esta profesión como un mecanismo de "defensa social". Ellas destacan que la figura y formación docente con que cuentan resulta ser de gran utilidad para protegerse, para proteger a su familia y comunidad. Por otra parte enfatizan la importancia que tiene para ellas su participación activa en la educación, ya que la consideran pieza clave en el mejoramiento de las condiciones de vida, tanto de niños como de adultos en sus respectivas localidades. (Howell, 1997, p. 266). Esto claramente sustenta la percepción de los investigadores, en el sentido de que las mujeres participantes en esta investigación definitivamente fueron quienes presentaron mayor y mejor 
información para los fines de esta investigación. Aún más allá, ellas presentaron propuestas de trabajo conjunto con instituciones de los Estados Unidos, esto con el objeto de lograr un mayor y mejor entendimiento.

\section{Métodos, técnicas, estrategias de investigación}

La entrevista directa y la observación en el aula fueron las principales estrategias metodológicas para la conducción de esta investigación, los investigadores seleccionaron a personal administrativo y profesores de secundaria y a aquellos de $5^{\circ}$ y $6^{\circ}$ de primaria. Eminentemente las instituciones fueron seleccionadas con base en su carácter público, ubicadas en contextos socioeconómicos de clase obrera. El hecho de seleccionar escuelas públicas estriba en que los alumnos, de clase baja, que regularmente atienden estas instituciones se encuentran en relativo aislamiento con lo que sucede en los Estados Unidos. Entrevistas frente a frente y discusiones grupales fueron efectuadas contando con la participación de veintisiete profesores de ciencias sociales y personal administrativo perteneciente a ambas escuelas.

Aunque en algunos casos hubo preguntas extra, las entrevistas incluyeron las siguientes preguntas:

- ¿Existe facilidad y apertura al dialogo en su clase?

- ¿Son los asuntos internacionales considerados como tópicos importantes a discutirse en su clase?

- Dentro de sus discusiones en clase, ¿es importante la guerra promovida por los Estados Unidos en Irak?

- Considera importante promover el análisis de la política Norteamericana (por ejemplo, la administración Bush)?

- ¿Qué percepciones en general han manifestado sus alumnos en relación con la política derivada de la administración Bush (por ejemplo, la guerra y la ocupación actual en Irak por tropas de los EU, la intromisión en Afganistán, etc.)?

- En caso de existir, ¿cuál es la diferencia ideológica entre México y los Estados Unidos más recurrente en las discusiones de clase?

- ¿Cuál es la opinión de la actual guerra contra el terrorismo? ¿Qué tipo de posiciones se comparten en este sentido en las discusiones en clase? 
- ¿Creé usted que la sociedad norteamericana debería considerar las opiniones generadas en las clases de ciencias sociales impartidas en aulas chihuahuenses?

La dinámica de entrevista-observación empleada por los investigadores se concentró en el cuestionamiento de los participantes, así como en la conducción de observación sistemática en algunos salones de clase a los que se tuvo acceso en ambas instituciones educativas. Además de la información obtenida a raíz de la respuesta de los profesores a las preguntas expresas, los investigadores desarrollaron explicaciones interpretativas tanto de los ambientes escolares como de documentos proporcionados por los mismos participantes. La metodología estuvo sustentada en las guías de la investigación cualitativa sugerida por Lincoln and Guba (1985), a través de la entrevista directa. Consecuentemente el énfasis del estudio se ubicó en la identificación de la estrategia con que se enseña, se discute y se trata el tema de la guerra promovida por los Estados Unidos en Irak en estas dos escuelas mexicanas. Esta modalidad naturalista de investigación pretende generar perspectivas concernientes a la "realidad de la hegemonía norteamericana y su autoabsorbida cultura dominante" (Young, 2002, p. 291).

Para fines de confiabilidad, las interpretaciones de las respuestas de los participantes, eminentemente docentes, fueron clarificadas por parafraseo o reformulación, esto mayormente cuando opiniones o réplicas a preguntas explícitas surgían tanto de la entrevista frente a frente como del resultado de la interacción, discusión y dinámica grupal.

Las entrevistas fueron conducidas en el idioma español, y dado que uno de los investigadores es hablante de esta lengua, su función resultó significativa en la labor de traducción e interpretación de las connotaciones y percepciones de los también participantes de habla hispana. Cabe mencionar, que el contar con un investigador hispano fue también útil para la identificación del lenguaje no verbal y del contexto cultural en que se dio la investigación.

La técnica de comparación (Glaser and Strauss, 1967) fue utilizada para la generación sistemática teórica. En primer lugar los investigadores ubicaron categorías de división instruccional y compararon los conceptos por incluirse en cada categoría. Las categorías principales fueron las siguientes:

(1) Tiempo instruccional y énfasis curricular sobre asuntos internacionales,

(2) énfasis curricular sobre política exterior norteamericana, y específicamente la Guerra de Estados Unidos en Irak, 
(3) las perspectivas oficiales de gobierno en la actual política exterior de los Estados Unidos,

(4) la perspectiva de administrativos y docentes acerca de la actual política exterior de los Estados Unidos, y en particular la guerra promovida por los Estados Unidos en Irak,

(5) las contribuciones de los alumnos en la discusión acerca de la guerra en Irak, y

(6) el tono general del diálogo dentro de los salones de ciencias sociales durante las discusiones de la guerra en Irak.

La teoría de sustento de la investigación se correlacionó con el análisis final obtenido una vez concluida la fase de entrevistas, esencialmente para asegurar algún tipo de clarificaciones o bien para identificar posibles inconsistencias sobre las interpretaciones de la investigación. Estos esfuerzos, o revisión de respuestas, fueron conducidos nuevamente con los mismos participantes a través de dialogo informal. Para triangulación (Pitman and Maxwell, 1992), los investigadores consultaron múltiples fuentes y tipos de información, básicamente a través de la investigación documental y observación directa, ya fuera para apoyar o contradecir lo previamente expuesto por los participantes. Esta verificación suministró un gran nivel de reforzamiento para el trabajo teórico de soporte de la investigación. Hay que destacar que los investigadores retornaron a las dos instituciones chihuahuenses en dos ocasiones, seis y nueve meses después respectivamente, después de la reunión inicial en la cual se recabó la información. Estas visitas estuvieron enfocadas a la confirmación de la información previamente recabada, para tal efecto, tanto observaciones como seguimiento a las entrevistas personales fueron nuevamente conducidas. Asimismo se desarrollaron discusiones generales en los grupos tratando de analizar respuestas de los mismos participantes originales, así como una nueva sesión de entrevistas para personal recién llegado.

Una vez más, la presencia de un chihuahuense desempeñándose como investigador incrementó los niveles de confianza con los participantes, lo cual permitió una mayor apertura de los canales de comunicación. En definitiva, este estudio no hubiese prosperado si a los participantes no se les hubiera explicado en sus propios términos los beneficios y alcances de la investigación en su propia lengua. Gracias a la confianza conseguida, un cúmulo significativo de información histórica y sociológica, documentos y narraciones fueron facilitados por parte del personal para ampliar la comprensión de la información obtenida. Básicamente esta apertura y cooperación permitió la identificación de la filosofía educativa 
de la institución, sus reconocimientos a la labor educativa y su plan institucional prospectivo. Debemos asimismo destacar que inclusive la cooperación llegó a tal grado que los mismos administradores asistieron la investigación definiendo los horarios de entrevista, abriendo las puertas de la escuela para efectuar observaciones directas en clases en proceso y contestando en todo momento a preguntas generales formales e informales.

Los investigadores extremaron precauciones para evitar el efecto de la "percepción inmaculada". Previo al estudio, los investigadores no habían tenido contacto de ningún tipo con los participantes. Los profesores que consintieron en participar firmaron su aceptación, lo cual entre otras cosas aseguró la confidencialidad de sus respuestas. El anonimato de la escuela será asimismo mantenido como confidencial. La participación fue en todo momento voluntaria y a los participantes se les hizo saber que podrían dejar de responder en el momento y por las causas que consideraran pertinentes.

Un profundo sentimiento de preocupación y cierta desconfianza por parte de los profesores y administrativos fue evidente al arribo de los investigadores. Este sentimiento se fue desvaneciendo tan pronto como se fueron enterando de que uno de los investigadores era chihuahuense, lo cual permitió una comunicación óptima. Lógicamente, para llegar a este nivel de distensión, la plática informal fue promovida con el fin de que ellos fueran los que iniciaran presentado sus dudas. La confianza de los participantes en los investigadores fue elemento clave que permitió acceder a los sentimientos verdaderos de los participantes. Cabe mencionar que para algunos de ellos esta era la primera vez que interactuaban con personal académico de los Estados Unidos.

\section{Análisis de resultados}

La planta docente y administrativa respondió eficazmente a cuestionamientos relativos a la pedagogía utilizada en las ciencias sociales, en especial acerca de su estrategia para abordar y discutir la política estadounidense en el salón de clase. Las técnicas de enseñanza reportadas fueron muy variadas, desde la tradicional cátedra unidireccional hasta el cuestionamiento directo a estudiantes mediante preguntas abiertas y debate acerca de estos asuntos de política internacional. Lo anterior, sin embargo, permitió obtener algunos patrones generales relativos al nivel de interés promovido en clase sobre los asuntos de los Estados Unidos. En la mayoría de los casos, los profesores reportaron que los alumnos están usualmente dispuestos a discutir este tipo de asuntos tan pronto como se les brinda la oportunidad de compartir su opinión. 
Del total de veintidós docentes entrevistados, cabe mencionar que 14 eran del sexo femenino. En ellas el concepto de "defensa" mencionado anteriormente y destacado por Howell (1997, p. 266) apareció en los resultados vertidos. Específicamente cuando algunas de ellas enfatizaron su "natural tendencia" a la creación de relaciones casi familiares con sus alumnos. Entremezclados con su obligación docente mencionan, se encuentran los sentimientos matriarcales, que con base en sus comentarios hacen que la relación profesora-alumno sea muy similar a la de madre-hijo. Este tipo de relación con alto grado de confianza es utilizada, entre otros, para "defender" a los alumnos de las influencias negativas provenientes de los medios y cultura norteamericanos. Incluso una de las entrevistadas expresó su sentida necesidad de defender a los estudiantes y sus frustraciones personales derivadas de la hegemonia estadounidense asumiendo que "Ios Estados Unidos del Norte es el enemigo del mundo."

En directa dependencia a sus específicos diseños curriculares, los profesores reportaron una variable cantidad de tiempo destinado a abordar los asuntos internacionales de los Estados Unidos. Hay que resaltar que todos los profesores y administrativos indicaron que en sus respectivos salones de clase así como en otros espacios de expresión, los alumnos usualmente comparten sus opiniones sobre familiares que viven y trabajan en los Estados Unidos. De hecho, una cantidad significativa de estudiantes han tenido la oportunidad de vivir en los Estados Unidos en alguna ocasión. Invariablemente, cuando la democracia y la vida interna estadounidense entran a colación y se discuten, un número considerable de estudiantes presentan sus opiniones haciendo uso de la información que de primera mano es obtenida de sus familiares que viven en ese país. Algunos profesores evidenciaron que debido a que el plan curricular oficial está enfocado expresamente a México este contenido tenía obviamente alta prioridad, y que el diálogo acerca de los Estados Unidos regularmente se da una vez que los requerimientos curriculares formales han sido cubiertos. Aparentemente, la rigidez de la actuación docente en torno a cumplir con el currículo oficial fue manifiesta, por lo cual los asuntos norteamericanos eran estrictamente secundarios.

En el lado opuesto, otros miembros de la planta docente y administrativa comentaron que la historia comparativa y los estudios sociales eran vitales para la educación de los alumnos, a pesar de las exigencias del currículo oficial. Cabe resaltar que una de las docentes participantes fue más allá para enfatizar la importancia que para ella tiene el hecho de que los estudiantes desarrollen un juicio más informado acerca de las relaciones internacionales. 
En suma, la mayoría de las discusiones en las escuelas participantes se ubican en temas nacionales de México en primer lugar, pero este nivel de discusión eventualmente incluye a los Estados Unidos por causa del entretejido histórico, social y económico que comparten ambos países.

Cuando las y los docentes participantes reportaron acerca de las reacciones que presentan los alumnos en relación con eventos recientes de los Estados Unidos, incluyendo el manejo de la guerra en Irak, la ocupación actual de este país por la milicia estadounidense, y la intervención en Afganistán, las respuestas, mediante interpretación, más recurrentes fueron las siguientes:

- Los alumnos están informados (de la política estadounidense) gracias a la cobertura otorgada por los medios de comunicación.

- Ellos están particularmente preocupados (de las decisiones estadounidenses internacionales) por el impacto que pudieran tener en los altos precios y en los efectos en los estándares de vida.

- Muchos de los estudiantes están interesados (en el desarrollo de la guerra en Irak) debido a que miembros de su familia pertenecen a la milicia estadounidense.

Cuando se profundizó sobre las estrategias didácticas de promoción a la discusión, los educadores destacaron que el aprendizaje de los estudiantes cualitativamente mejora cuando son partícipes de la discusión de estos asuntos internacionales estadounidenses que de una $u$ otra forma tienen impacto en México. Asimismo concuerdan en afirmar que la argumentación en torno a la política estadounidense motiva en los alumnos la capacidad de liderazgo y responsabilidad. Hubo aceptación al afirmar que el incorporar este tipo de discusiones dentro del plan curricular motiva el aprendizaje, ya que las habilidades de comparación, análisis y reflexión son ampliamente ejercitadas con la "simple discusión". Otro de los profesores destacó que la educación del alumnado en esta modalidad didáctica debe necesariamente incluir la adopción de una "actitud propositiva" o una actitud de defensa sobre una posición u opinión personal en relación con cualquier tema, ya que esta es el fundamento estratégico básico para la acción reflexiva.

Asimismo, algunos educadores refirieron que la mayoría de los estudiantes desean que la violencia termine, ya que consideran la guerra en Irak como una expresión más de la política de "expansión e imperialismo" estadounidense en contra de personas consideradas diferentes por su orientación política y religiosa. Algunos perciben a los Estados Unidos en este sentido como la Roma contemporánea, bajo los problemas y presiones implícitos en su propio intento por controlar el mundo. 
Otra de las opiniones generalizadas en este sentido fue una sentida frase: "Si nos arrancáramos la piel, todos seríamos iguales." En otras palabras, los Estados Unidos como nación debe verse en el espejo y atender sus propios defectos. No hay raza superior ni inferior, simplemente distintas creencias, cosmovisiones y formas particulares de interpretar el mundo.

Cuando se preguntó acerca de otras temáticas alejadas de la guerra en Irak, que por su importancia son tratadas en clase, una amplia gama de elementos estadounidenses aparecieron en escena. Los temas son, en esencia, una crítica hacia una nación que se autodenomina como un modelo de equidad racial, como país líder en el control de la contaminación y calentamiento global, que está a la vanguardia en su preparación ante contingencias ambientales. Además, los profesores reportan inconsistencias presentes en la sociedad norteamericana, surgidas del debate y el cuestionamiento de los alumnos. Una de ellas destaca que los alumnos creen que atletas provenientes de estratos socioeconómicos marginados -principalmente afro americanos- son valorados socialmente solo por su capacidad atlética. Otra de las inconsistencias es la alta emisión de dióxido de carbono en sus ciudades, y aún así la renuencia a cumplir con los acuerdos de Kioto. Una más lo constituye la falta de atención oportuna a las víctimas del huracán Katrina en donde encuentran cierta práctica racista como explicación a la lentitud en la asistencia. Pero definitivamente existe un alto nivel de preocupación por la histórica discriminación en los Estados Unidos, ya que muchos de los alumnos, o bien miembros de su familia o conocidos la han padecido en carne propia y reportado de alguna manera. "Esta es la cara más cercana y conocida de los Estados Unidos".

Decía un expresidente mexicano..."pobre México, tan lejos de Dios y tan cerca de los Estados Unidos". Al parecer esta cercanía sigue siendo eminentemente geográfica, pues los 3.147 kilómetros de frontera mutua se percibe de manera generalizada como la entrada ilegal a la discriminación.

Algunos profesores y administrativos detectaron algún tipo de supersticiones y fatalismo detrás de las explicaciones de los estudiantes en su aparente competencia con los Estados Unidos. Consecuentemente, existe la creencia de que los Estados Unidos están siendo castigados por sus crímenes del pasado. Los alumnos explican dicho fenómeno aseverando que tanto en el pasado como en el presente la sociedad estadounidense sufre de una pérdida de moralidad. Ellos atribuyen muchos de los problemas como resultantes de la "falta de valores familiares" que definitivamente comparan con aquellos que las familias mexicanas tienen. Además, otros alumnos mencionan que a pesar de que las oportunidades 
son limitadas en México, los trabajadores inmigrantes son frecuentemente maltratados. Por esta razón es mejor permanecer en México por la "violencia, falta de respeto y criminalidad existente en los Estados Unidos".

Todos los educadores entrevistados están de acuerdo en que los medios de comunicación estadounidense describen a México desde una óptica unilateral y negativa. La mayoría de ellos expresaron opiniones en el sentido de la diferencia de opiniones entre ambos países no es algo nuevo, al respecto destacan que las escuelas estadounidenses no han enseñado nunca la verdadera realidad detrás de la invasión a México que resultó en los tratados de Guadalupe-Hidalgo en 1848 y la consecuente apropiación de tierra, gente y recursos.

En relación con acontecimientos más recientes, profesores y administrativos opinaron que los estudiantes estaban de acuerdo con la asistencia brindada por el gobierno mexicano a las víctimas del huracán Katrina. Sin embargo existe un sentimiento generalizado en el sentido de que poco reconocimiento tuvo esta acción, en que la gente y gobierno de México tendieron la mano al vecino del norte. Esto promovió la discusión en el salón de clase para dilucidar el porqué de esta falta de reconocimiento de parte de los Estados Unidos.

Los docentes entrevistados enfatizaron que los estudiantes tienden a generalizar explicaciones orientadas a comprender la estrategia detrás de la política exterior estadounidense. Destacan que el esquema económico impuesto por los Estados Unidos "llega a todos los confines del mundo". Agregan que la explicación a la actuación internacional de este país está solamente orientada por la apropiación petrolífera.

Pero además advierten que hay otros elementos internos que reclaman aún por respuestas concretas y que a la fecha ocupan los escenarios de debate. Los cuestionamientos que recurrentemente plantean los alumnos son los siguientes:

"¿Qué podemos decir de los Estados Unidos y la muerte de niños y gente inocente en Irak?"

“¿Por qué esta nación no gasta su dinero en otras causas (además de la guerra) como apoyar la disminución del hambre en África?"

“¿Por qué existe tanta diferencia en los pagos de salarios en compañías multinacionales en México, en relación con el pago por la misma compañía en los Estados Unidos?"

Después de los ataques del 11 de septiembre, los alumnos lógicamente se vieron bombardeados por los eventos televisivos. Los alumnos en general expresan su tristeza por las víctimas de los ataques terroristas en los Estados Unidos. A pesar de que la mayoría de 
los alumnos desaprueba los ataques terroristas, también afirman que existe una gran posibilidad de que los ataques continúen debido a la política externa de este país. En casi la totalidad de los alumnos entrevistados se pudo percibir un profundo deseo de asegurar la paz. Pero los profesores destacan que los Estados Unidos tienen una larga tradición de intervención militar, la cual en cierta forma promueve estos ataques terroristas como una reacción "natural" a la intromisión de este país en varias partes del mundo. Otros elementos promotores del debate en el aula resultaron ser el nacionalismo, la religión y la lucha por la hegemonía mundial considerados como elementos detonantes del terrorismo.

En conclusión, los temas centrales obtenidos mediante las entrevistas ubican a los Estados Unidos en una gran necesidad de autorreflexión, acción y reajuste de sus responsabilidades internacionales. Por consiguiente, los Estados Unidos deben reexaminar su política externa tendiente a mantener otros países pasivos y sin resistencia a sus esquemas de política externa. Con base en Freire (1970), los opresores se relacionan con oprimidos, a través de la subyugación, con el objeto de mantenerlos complacientes. Los Estados Unidos indiscutiblemente asumen este rol con sus vecinos continentales. Freire, en este mismo sentido destaca que:

Esta subyugación, sin embargo, no tiene que ver directamente con la gente, asimismo no requiere una comunicación verdadera. Esta dominación se da por parte del opresor mediante la difusión de mitos, considerados relevantes para el mantenimiento del estatus quo: por ejemplo, el mito consistente en mantener una "sociedad libre". (p. 39)

\section{Implicaciones}

Fue muy palpable la cordial aceptación de la comunidad escolar en ambos centros educativos para los investigadores, y en contraste fue lastimoso constatar que la cercanía geográfica no se ha traducido en una deseable cercanía educativa. Estos 350 kilómetros de distancia nos ubican prácticamente unidos en el mapa y extremadamente lejanos en la realidad. Es difícil concebir que respectivamente la inmigración ilegal sea la cara más conocida de México en Norteamérica y la discriminación la práctica corriente de los Estados Unidos para México.

El reconocer mediante la visita de los investigadores que para algunos de los participantes este era el primer acercamiento "cultural" binacional nos hizo remontar a todos hacia tiempos coloniales. Algunos de los profesores comentaron que en un principio pensaron que los investigadores habían planeado esta visita con el objeto de escrutinio y crítica de la labor de las plantas docentes y administrativas. Lo extraordinario es que a pesar 
de la evidente desinformación sobre la visita de los investigadores abrieron la puerta y la dejaron abierta con un dejo de esperanza hacia el futuro.

Una vez que plenamente identificaron los alcances de la investigación, ellos mismos expresaron su satisfacción por haber sido invitados a participar. Una de ellos expresó que la realimentación y la entrevista en parte la motivaron para fortalecer su práctica docente y su compromiso con la educación, algo muy importante a pesar del bajo salario que se recibe por esta función. Dada la necesidad derivada de una mayor y mejor comprensión de lo que pasa en ambos lados de la frontera, la pregunta obligada para ellos fue que cómo consideraban conveniente proceder para un mejor entendimiento binacional, con objetivos educativos mutuos que reduzcan generalizaciones infundadas.

La respuesta consistió en la necesidad de promover un mayor número de intercambios tanto de profesores como de alumnos. El apoyo compartido, según su opinión debe darse en forma bidireccional para lograr el conocimiento de la lengua y de otros rasgos culturales propios de ambos países.

Se pudo advertir que los profesores mexicanos apoyan la idea de una mayor cooperación entre México y los Estados Unidos; sin embargo, expresan preocupación por la falta de crédito y reconocimiento a la labor y cooperación de México para con los Estados Unidos en varios aspectos a través de la historia, que se han dado tanto de manera formal como informal. En otras palabras existe el sentimiento de que la percepción estadounidense se centra únicamente en los aspectos negativos de la vida social mexicana; por ello, México es usualmente descrito de forma negativa tanto por los medios como por el gobierno norteamericano.

Los docentes y administrativos de las escuelas entrevistados expresaron un verdadero interés por incrementar el conocimiento de la vida"más allá de las fronteras de México". En este sentido, ellos plantean que el gobierno de México debe proponer estrategias promotoras de una más positiva imagen de lo que verdaderamente es la educación, así como la gente de México. Para esto, deben necesariamente existir esfuerzos conjuntos enfocados al mejoramiento del sistema educativo, en lugar del propio y "único esfuerzo del gobierno mexicano por mantenerse en el poder". Los entrevistados adoptaron posiciones tales como, "las necesidades de nuestra gente están en un completo segundo plano," y "el gobierno necesita destinar más recursos para apoyar a las comunidades rurales y su calidad de vida."

Evidentemente, ambos lados de la frontera pueden beneficiarse al contar con intercambios educativos. Rippberger y Staudt defienden la idea consistente en que los 
profesores norteamericanos pueden reflexionar sobre su propio sistema educativo mediante la observación de los siguientes aspectos presentes en la educación mexicana:

Los planes curriculares estadounidense y mexicano comparten un gran número de valores comunes como la valentía, perseverancia, y responsabilidad. Valores colectivos, como la solidaridad, patriotismo, amistad, y generosidad que aparece más fuerte en México tanto como los temas de autoridad, respeto y orden. (Rippberger \& Staudt, 2003, p. 66)

Definitivamente, docentes y administrativos pertenecientes tanto a México como a los Estados Unidos se beneficiarían a raíz de una mejor comprensión acerca de los roles de docentes y alumnos de la contraparte, los beneficios serían mutuos ya que ambos conforman una comunidad binacional. Los educadores deben analizar aquellas políticas que de alguna forma nos separan y entender mejor los "impedimentos establecidos por los sistemas políticos y educativos que limitan o impiden el intercambio de experiencias en educación" (Hampton, Liguori, Rippberger, 2003, p. 9).

A pesar de la disposición existente para efectuar intercambios culturales de largo alcance, es evidente que las escuelas mexicanas padecen la carencia de financiamiento, razón por la cual un impedimento fuerte está constituido por la inexistencia de fondos para un eficaz intercambio de profesores, administrativos y alumnos. El personal administrativo en Chihuahua ha expresado su interés en la promoción de intercambios culturales con la intención, entre otras, de mejorar su tecnología educativa, diccionarios y material bilingüe, equipo adicional básico como videograbadoras y materiales para equipamiento de laboratorios. Lamentablemente es imperativo reconocer que a la fecha no existe una estrategia seria que visualice esta pretensión al menos en el corto plazo.

Las conclusiones específicas de este estudio indican que el gobierno de los Estados Unidos debe remover su ostracismo y aprender de las opiniones de otros, incluyendo por supuesto a los educadores y estudiantes chihuahuenses. Las opiniones en materia histórica, política y social fuera de los Estados Unidos pueden generar una mayor riqueza para el conocimiento de las perspectivas dominantes de este país. Tal y como lo dice Young (2002) la cultura norteamericana dominante es, y debe ser continuamente analizada, porque, a pesar de su retórica de superioridad "tanto dentro como fuera del país; los Estados Unidos no es excepcional, solo es excepcionalmente poderoso" (p. 291).

Dentro de los alcances de este estudio, los investigadores establecen recomendaciones en las que se enfatiza la necesidad de crear puentes de entendimiento en 
un tiempo en el que al parecer los muros van ganando la batalla. Las entrevistas estuvieron enfocadas a la captura de los sentimientos reales tanto de las mujeres, como de los hombres participantes, mismos que voluntariamente compartieron sus más sentidos sentimientos con relación al tema de estudio.

Uno de los profesores sintetizó con su comentario el sentimiento generalizado de los educadores chihuahuenses hacia la política exterior estadounidense al usar la universalmente conocida frase del Presidente Benito Juárez: "Entre los individuos, como entre las naciones, el respecto al derecho ajeno es la paz". Es evidente que los educadores estadounidenses tienen mucho que perder al no contravenir la cultura del dominio que los engulle y por otro lado mucho que ganar a través de los intercambios culturales con su contraparte mexicana que solo se encuentra a un río de distancia, un río que es cierto; es grande y es bravo a la misma vez, tanto que ha demostrado ser incapaz de ser cruzado con puentes de entendimiento. 


\section{Referencias}

Andrade de Herrara, Victoria. (1996). Education in Mexico: Historical and contemporary educational systems. In J.L. Flores (Ed. \& Trans.), Children of the frontera: Binational efforts to serve Mexican migrant and immigrant students. Recuperado el 16 de setiembre 2006, en base de datos ERIC.

Erickson, Ralph . (2002). Foundations of the Mexican federal educational system. The Clearing House, 75 (3), 146-150.

Freire, Paulo. (1970). Pedagogy of the oppressed. New York: Continuum.

Glaser, Barney G., \& Strauss, Anselm L. (1967). The discovery of grounded theory: Strategies for qualitative research. Chicago: Aldine.

Hampton, Elaine, Ligouri, Olga, \& Rippberger, Susan. (2003). Binational border collaboration in teacher education. Multicultural Education 11 (1), 2-10.

Instituto Nacional de Estadística, Geografía e Informática - INEGI. (2000). Mexican National Statistics, Geographic and Informatics Institute. Recuperado el 20 de febrero 2006, http://www.inegi.gob.mx/est/contenidos/espanol/rutinas/ept.asp?t=mpob93\&c=3839\& $\underline{\mathrm{e}=08}$

Howell, Jayne. (1997). This job is harder than it looks: Rural Oaxacan women explain why they became teachers. Anthropology \& Education Quarterly, 28(2), 251-279.

Jelin, Elizabeth. (1990). Women and Social Change in Latin America. London: Zed Books.

Levinson, Bradley A. (1998). Student culture and the contradictions of equality at a Mexican secondary school. Anthropology \& Education Quarterly, 29(3), 267-296.

Lincoln, Yvonna S. \& Guba, Egon G. (1985). Naturalistic inquiry. Beverly Hills, CA: Sage.

Pérez Pascual, Rafael. (2006). La educación en el México del año 2000. Recuperado el 15 de febrero 2006, de http://www.rolandocordera.org.mx/confi/perez.htm

Pescador Osuna, Jose Angel. (1985). Mexico: System of education. In Torsten Husen, (Ed.) The International Encyclopedia of Education, 6. New York: Pergamon Press.

Pitman, Mary A. \& Maxwell, Joseph A. (1992). Qualitative approaches to evaluation: Models and methods. In LeCompte, M. D., Millroy, W.L., \& Preissle, J. (Eds.).The Handbook of Qualitative Research in Education. San Diego, CA: Academic Press.

Program for Educational Modernization. (1989-1994). México, D.F.: Secretaria de Educación Pública.

Ríos Morales, Manuel \& Caballero, Juan Julián. (2002). Perspectives on the formation of indigenous teachers for an intercultural teacher education in Oaxaca, Mexico. Action in Teacher Education, 24 (2). 55-63. 
Rippberger, Susan J. \& Staudt, Kathleen. (2003). Pledging allegiance: Learning nationalism at the EI Paso-Juarez border. New York: Routledge Falmer.

Sistema Educativo de los Estados Unidos Mexicanos Principales Cifras Ciclo Escolar. (2004). Secretaría de Educación Pública, Subsecretaría de Planeación y Coordinación. Dirección General de Planeación, Programación y Presupuesto. Recuperado el 15 de febrero de http://www.sep.gob.mx/work/appsite/princif2003/Princcif2003.pdf.

Torres-Bodet, Jaime. (1961). Remarks by Jaime Torres Bodet about ILV. Recuperado el 19 de febrero 2006, de http://www.sil.org/mexico/ilv/iTorresBodet.htm

Wilcox, Brad \& Moreno, Monica Vidal. (1999). Turning points in Mexico. Childhood Education, 75(6), 359-362. 